\title{
Repérage, description et conservation des variétés fruitières régionales
}

\author{
R Bernhard ${ }^{1}$, E Leterme $^{2}$, L Olivier ${ }^{3}$, MF Tarbouriech ${ }^{4}$
}

1 Institut national de la recherche agronomique, station de recherches fruitières, BP 81, 33883 Villenave-d'Ornon Cedex;

2 Groupe de ressources phytogénétique d'Aquitaine, Écomusée, 40630 Sabres;

3 Conservatoire botanique national de Porquerolles, rue Sainte-Claire, 83400 Hyères;

4 Conservatoire botanique national alpin de Gap-Charance, Domaine de Charance, 05000 Gap, France

\begin{abstract}
Résumé - Le repérage des variétés fruitières régionales, leur conservation et leur description sont 3 objectifs, qui logiquement devraient constituer 3 phases successives; ils sont en réalité le plus souvent poursuivis simultanément. Du fait de leur rareté et de la méconnaissance qui les entourent pour la plupart d'entre elles, les variétés anciennes locales se trouvent souvent confondues à des variétés ayant perdu leur identité mais introduites à partir d'autres régions ou d'autres pays. Leur comportement agronomique et leurs caractéristiques génétiques sont totalement inconnus. Le repérage des variétés locales historiquement connues, et parfois décrites, doit être associé à la prospection de variétés locales de noms inconnus (souvent hybrides naturels) remarqués pour telle ou telle caractéristique agronomique favorable à l'arboriculture. La description du matériel végétal repéré, qui peut commencer par plusieurs visites des arbres originaux disséminés, nécessite la création de vergers de rassemblement correctement cultivés et systématiquement observés (avec des techniques et des méthodes rigoureuses et normalisées). Ces vergers indispensables à l'effort global de conservation ne constituent pas encore de véritables conservatoires. La conservation des variétés fruitières nous semble devoir être réalisée prioritairement par l'implantation, en nombre limité, et dans les principales grandes régions fruitières de véritables vergers conservatoires parfaitement cultivés, et gérés par des structures le plus pérennes possible; chaque conservatoire devrait concentrer son activité sur le matériel génétique essentiellement local et bien identifié. Au titre de la sauvegarde, le double de ce matériel devrait être situé dans des collections d'amateurs bénévoles ou autre structure (communes, écomusées, lycées agricoles...) et, éventuellement dans un laboratoire équipé pour la cryoconservation de greffons, pollen, semences... La priorité accordée à la réalisation des vergers conservatoires est liée à l'intérêt génétique (mise à disposition des chercheurs d'ovules, de pollen ou autre matériel), culturel (patrimoine local) ou économique (diversification agricole) du matériel végétal recensé.
\end{abstract}


Summary - Prospection, characterisation and conservation of local fruit tree varieties. Local fruit tree varieties (and also interesting natural seedlings) should be studied with respect to different climates and different parts of the country. Good characterisation of each variety needs systematic observation in the same cultural conditions and comparison with the well-known varieties. After these 2 steps, several conservatories or repository orchards of local varieties should be planted in each major historical fruit-growing district. This would be of value to local people and growers of traditional varieties, maintain a natural evolution (seedlings and mutations), and facilitate breeding work (permanent availability of ovules and pollen). In situ conservation and cryoconservation could be secondary methods of safeguarding fruit tree diversity.

fruit tree / genetic diversity / conservation

\section{INTRODUCTION}

L'amélioration des plantes pour toutes les espèces nécessite le rassemblement de nombreuses variétés, la sélection des meilleures, puis dans un deuxième temps par hybridation le rassemblement chez un même génotype de caractères intéressants disséminés dans plusieurs autres.

Pour les arbres fruitiers, ce n'est qu'assez tardivement, en France, que la recherche d'État a commencé à réaliser ce travail de rassemblement en un même lieu et de caractérisation des variétés : 1939 pour 2 espèces à noyaux, 1950-60 pour les espèces à pépins et les autres espèces à noyaux. Le maintien de ces collections variétales encombrantes a posé de nombreux problèmes, leur intérêt n'a pas toujours été bien compris et à l'exception de 2 espèces (pêcher et prunier) la publication de catalogues descriptifs des variétés étudiées n'a pas été réalisée; les éléments existent mais dorment dans de nombreux classeurs et cahiers d'observation. À défaut d'un travail de systématique moderne, on vient de rééditer les 6 volumes du dictionnaire pomologique qu'André Leroy, pépiniériste, a publié en 1867! Remarquable travail de description des fruits, auquel il manque cependant des informations morphologiques et physiologiques concernant l'arbre, et qui ne concerne qu'un certain nombre de variétés. De plus il existe actuellement des méthodes d'étude génétique.

La lassitude amenée par une excessive standardisation des variétés de pommes et de poires, la qualité gustative insuffisante des fruits commercialisés souvent mal cultivés (plantations trop denses, récoltes trop immatures, etc) et l'intérêt porté aux variétés traditionnellement cultivées ont contribué à la création continuelle depuis 1975 de nombreuses associations dont le but est d'organiser le sauvetage d'un patrimoine local ancien ou de créer des centres de ressources génétiques régionaux... Ces associations et organismes effectuent des travaux très lourds à mener : prospection, opérations de greffage, constitution et entretien de verger conservatoire, observations, description variétale, constitution de photothèques, expositions fruitières attirant des foules parmi lesquelles un grand nombre d'amateurs et chercheurs.

L'Association française pour conservation des espèces végétales (AFCEV) groupement d'intérêt scientifique, a constitué le groupe de travail «arbres fruitiers» dans le but de coordonner les activités publiques, associatives ou privées, concernant la conservation des variétés fruitières : prospection, étude, sauvegarde, voire relance 
des anciennes variétés fruitières. Son but est de suggérer des méthodes de travail rigoureuses conduisant à terme à un système de conservation fiable et régionalisé, le moins onéreux possible et constituant des ressources génétiques disponibles pour l'amélioration des plantes (fig 1).

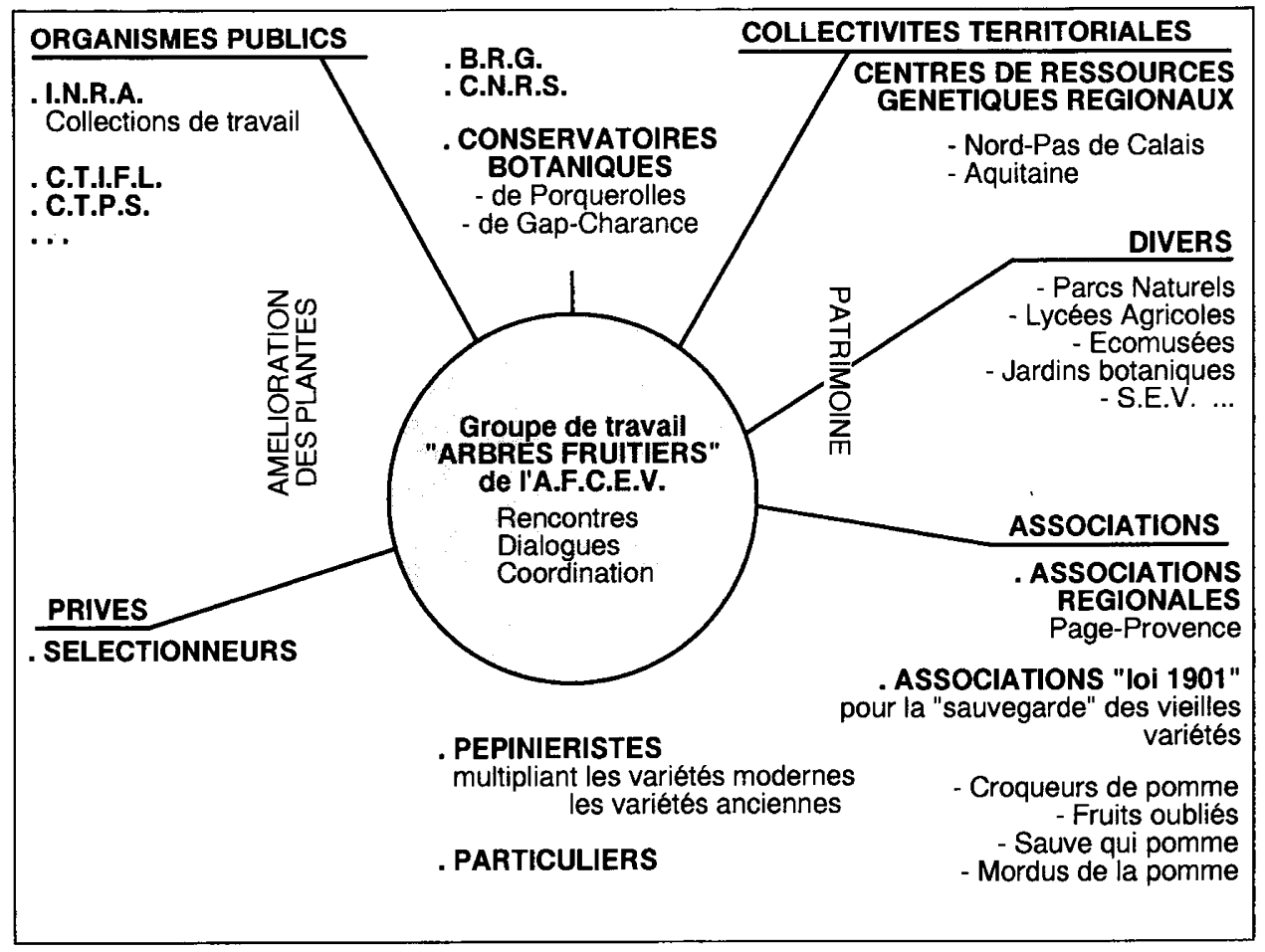

Fig 1. Fonctionnement du groupe de travail «arbres fruitiers» de l'AFCEV.

\section{CONSERVATION DE LA VARIABILITÉ FRUITIÈRE}

Le souci de conservation d'une variabilité fruitière étendue n'est pas nouveau ; des collections existent dans les stations de recherches, dans certains établissements d'enseignement, chez quelques pépiniéristes créateurs de variétés; mais dans ces collections la proportion de variétés indigènes s'est beaucoup réduite au bénéfice de cultivars étrangers déjà améliorés. Ce qui est nouveau, c'est le désir de faire (ou de refaire plus à fond) un inventaire de cultivars locaux anciens, même quand leur productivité ou leurs caractéristiques commerciales ne sont pas toujours remarquables, de les rassembler localement de les montrer au public et éventuellement de relancer la culture de quelques-unes au moins localement.

Même si les motivations des différents membres concernant le repérage, la description, la conservation, voire la relance de la culture de variétés régionales 
sont différentes, l'AFCEV organise un minimum de coordination et de rigueur dans le travail, afin que la pluralité des actions constitue un élément non négligeable de sécurité pour la conservation de la diversité.

Repérage, rassemblement puis conservation, description et éventuellement valorisation des ressources génétiques fruitières indigènes devraient logiquement constituer des phases successives; en fait elles sont le plus souvent menées de pair. Une description précise nécessite d'observer des arbres d'âge identique ou très voisins greffés sur même porte-greffes; placés dans une parcelle homogène avec présence de témoins. La conservation proprement dite ne devrait intéresser que les variétés locales après élimination des variétes synonymes ou celles identifiées comme étrangères ou de régions très éloignées et conservées par ailleurs.

\section{REPÉRAGE}

Premièrement, il s'agit généralement de retrouver grâce à des prospections systématiques des variétés historiquement connues citées dans la bibliographie et généralement décrite de façon très sommaire. S'il reste encore plusieurs individus d'une même variété l'introduction en verger d'étude devrait se limiter aux 2 ou 3 clones apparemment les plus performants et les plus sains. Ces arbres sont souvent âgés et situés à proximité d'anciennes fermes, dans des jardins ouvriers, parc de château...

Deuxièmement il s'agit aussi du repérage d'individus dénommés localement, et présentant un intérêt particulier concernant l'aspect, le goût, l'époque de maturité, la forme particulière de l'arbre, la résistance à un parasitisme ou à un accident climatique, etc.

Ces individus peuvent être une variété originale issus de graine et multipliée par la suite à cause de son intérêt ou une introduction ancienne d'un autre pays ou d'une région éloignée.

Pour ce stade de repérage, urgent et très important, toutes les méthodes de prospection peuvent être envisagées (enquête dans les mairies, sur les marchés locaux, avis de recherche par voie de presse, expositions, concours, etc).

Sous l'égide de l'AFCEV, du Bureau des ressources génétiques et de l'Association régionale PAGE PACA, un ouvrage intitulé $A$ la recherche des variétés locales de plantes cultivées (1986) a été écrit par Philippe Marchenay et Marie-France Lagarde.

Il donne toutes les indications utiles pour la préparation et la conduite des prospections. Un chapitre est plus particulièrement consacré à l'organisation des prospections et un autre à la collecte du matériel végétal et des savoirs populaires qui sont liés à ces variétés.

L'AFCEV recommande la réalisation de ces prospections pendant 2 à 3 saisons consécutives avec plusieurs visites des individus repérés et marqués avant la constitution d'un verger de rassemblement et d'étude. Pour des raisons de sécurité bien compréhensibles, tout individu repéré au cours des différentes prospections devrait être systématiquement introduit en pépinière d'attente et une grande rigueur dans le clonage de chaque individu retenu devrait être réalisée (tenue d'un registre d'introduction, étiquetages assortis par sécurité de plans très précis, etc). 


\section{DESCRIPTION}

La description des caractéristiques morphologiques et physiologiques des types variétaux rassemblés en verger d'étude est la phase la plus difficile à réaliser car elle nécessite un travail presque continu pendant plusieurs saisons successives.

Si une première description sommaire et peu rigoureuse peut être faite à partir des arbres originaux disséminés dans la nature, ce n'est que dans un même verger et en comparaison avec des variétés clonales témoins que l'on peut réaliser la description, en particulier pour les caractères physiologiques (phénologie, sensibilité aux accidents et parasites...). Il est naturellement souhaitable que les arbres de ces vergers d'étude soient implantés dans des conditions les plus semblables possibles (âge et porte-greffes identiques, conduite et façons culturales homogènes, etc).

L'AFCEV, avec l'aide du ministère de la Recherche, a mis au point les outils nécessaires à la description des cultivars retrouvés et ceci pour chaque espèce. Des fiches standard ont été éditées et diffusées auprès des associations, conservatoires, centres de ressources... Un guide d'utilisation de ces fiches a été également réalisé et diffusé; des réunions d'information et de formation sont organisées. Ces fiches et mode d'emploi concernent les espèces pommier, poirier, pêcher, prunier, cerisier, figuier et olivier. La fiche châtaignier est en préparation. À titre indicatif, est présenté ci-après le document Description pomologique des pêchers (annexes 1 et 2).

\section{CONSERVATION}

La conservation des ressources génétiques peut théoriquement se réaliser de différentes manières (sous forme de conservation des graines, de cryoconservation des greffons, de vitroculture...). Cependant, plusieurs raisons militent en faveur de la réalisation de vergers conservatoires :

- la nécessité de décrire en plantations comparatives ces variétés qui sont peu connues; pour pouvoir utiliser le matériel végétal à des fins scientifiques (génétiques) ou économiques, il faut le connaître (donc l'étudier et le décrire) et l'avoir rapidement à disposition;

- les techniques de conservation en laboratoire ont un certain nombre d'inconvénients; la vitroculture, faisant se succéder rapidement les générations, est l'occasion de mutations qui modifient les caractères du végétal; pour la cryoconservation de bourgeons, se pose le problème de la régénération des plants... dans ces cas-là, le matériel végétal n'est pas disponible pour une utilisation immédiate;

- limitation des risques de perte en conservant les variétés dans les régions où elles sont bien adaptées aux conditions pédo-climatiques;

- les personnes qui ont pris l'initiative de la recherche de cultivars anciens veulent pouvoir étudier le patrimoine régional qu'ils ont contribué à réunir et le montrer, seul moyen de le faire connaître et de sensibiliser le grand public à l'intérêt de la conservation;

- l'observation du comportement de certaines variétés intéressant les amateurs est un élément de promotion;

- la nécessité, du moins en théorie, de ne pas bloquer l'évolution, stimulée par le fait que chaque année dans les vergers conservatoires, des graines pourraient être produites en fécondation croisée, libre ou contrôlée; 
- la possibilité enfin dans ces conservatoires de disposer rapidement et en abondance de greffons, ovaires réceptifs et pollens, ce qui facilitera grandement le travail des créateurs de nouvelles variétés.

Ces vrais vergers conservatoires, en nombre forcément limité, devraient être installés dans chacune des régions les plus concernées par les fruitiers où n'y seraient maintenus que les cultivars anciens de la région considérée plus les quelques écotypes prospectés (issus de semis) mais présentant une caractéristique intéressante (et aussi quelques variétés modernes servant de repères et de témoins en particulier celles résistant à la tavelure).

L'AFCEV et le Bureau des ressources génétiques ont réalisé un document de référence pour la constitution de tels vergers conservatoires. Ce document, intitulé «Cahier des charges pour la constitution des vergers de conservation de variétés locales ou anciennes d'espèces fruitières», définit toutes les phases de la constitution d'un verger conservatoire, les conditions d'évaluation et de notation des arbres, les témoins à planter espèces fruitières par espèces fruitières. Les éléments nécessaires à l'attribution d'un numéro national d'identification des arbres en collection sont aussi fournis.

L'expression «verger conservatoire» a souvent été utilisée à tort pour des vergers de rassemblement ou d'étude participant au sauvetage et à la conservation des anciens cultivars menacés de disparition. On a aussi utilisé à tort ce terme de conservatoire pour les vergers constitués de pieds mères authentiques et sains (virus free) servant de base à la certification génétique et sanitaire et n'intéressant que les seules variétés de grande culture.

Il nous a paru jusqu'ici inutile et trop onéreux de concevoir des conservatoires de notre diversité fruitière avec des individus virus free; en effet une thermothérapie, opération très coûteuse, peut si nécessaire être appliquée a posteriori pour les quelques variétés dont on envisagerait la multiplication sur une certaine échelle. Il faut par ailleurs remarquer que de nombreux virus ne sont pas transmis lors de la reproduction sexuée.

Cependant, pour les arbres fruitiers à noyaux, l'introduction et le développement relativement récent d'un virus particulièrement nocif, la Sharka, va obliger, principalement pour les espèces abricotier, pêcher, prunier, à compléter les dispositifs existants par la création d'un conservatoire particulier dans un site très isolé où seul un matériel végétal sain et parfaitement contrôlé pourra y être introduit et surveillé. (La Sharka affecte l'aspect et la qualité des fruits; elle est transmise par plusieurs espèces de pucerons et peut se maintenir dans l'environnement, non seulement sur des pruniers sauvages, mais aussi sur quelques plantes herbacées).

Un tel dispositif viendrait compléter ce qui a déjà été réalisé pour les fruitiers à pépins dans les Hautes-Alpes (Conservatoire botanique national alpin de GapCharance) par la mise à l'abri des contaminations par le feu bactérien (Erwinia amylovora) de l'essentiel des collections nationales de poiriers et de pommiers.

Cependant, à l'heure actuelle, les variétés fruitières anciennes sont étudiées dans des vergers de rassemblement qui ont reçu l'agrément de l'AFCEV lorsqu'ils sont correctement conçus et très peu d'entre eux ont une structure conservatoire capable d'assumer la pérennité des collections. La plupart des variétés rassemblées ne sont pas étudiées et restent encore dans des vergers de collections qui n'ont pas pu recevoir l'agrément de l'AFCEV. 
Le groupe de travail «arbres fruitiers» de l'AFCEV travaille actuellement sur l'établissement d'un fichier national pour connaître ce qui est conservé en France (fig 2).

\begin{tabular}{|c|c|}
\hline $\begin{array}{l}\text { QUATRE ETAPES POUR LA CONSERVATION } \\
\text { DES VARIETES FRUITIERES }\end{array}$ & PUBLICATIONS DEI' AFCEV \\
\hline $\begin{array}{l}\text { REPERAGE DES VARIETES" } \\
\text { - prospection ( } 2 / 3 \text { du territoire français ?) } \\
\text { - enquetes } \\
\text { - recherche (bibliographie, archives...) }\end{array}$ & $\begin{array}{l}\text { "A la recherche des variétés locales de plantes } \\
\text { cultivees". M.F. LAGARDE - } 1986 \\
\text { AFCEV-BRG-PAGE PACA }\end{array}$ \\
\hline $\begin{array}{l}\text { DESCRIPTION } \\
\text { - des varietes, in-situ ou ex-situ; } \\
\text { - effectuées par differents partenaires: associations, } \\
\text { centre de ressources genetiques, conservatoire } \\
\text { botanique.... } \\
\text { - formation des intervenants; } \\
\text { - confrontation aux données bibliographiques. }\end{array}$ & $\begin{array}{l}\text { "Fiches de description fruitieres" POMMIER, } \\
\text { POIRIER, PECHER, PRUNIER, CERISIER, } \\
\text { FIGUIER, OLIVIER... } \\
\text { + "Guide méthodologique d'utilisation" } \\
\text { AFCEV-MRT }\end{array}$ \\
\hline $\begin{array}{l}\text { CONSERVATION } \\
\text { - sous forme de vergers, dans les régions; } \\
\text { - meilleure adaptation aux conditions de milieu; } \\
\text { - interet culturel local (plus mobilisateur) } \\
\text { - expertise de } 22 \text { collections ( } 7 \text { ont éte agrées, } 10 \text { sont } \\
\text { importantes). }\end{array}$ & $\begin{array}{l}\text { "Cahier des charges pour la constitution d'un } \\
\text { verger conservatoire" } \\
\text { AFCEV }\end{array}$ \\
\hline $\begin{array}{l}\text { VALORISATION } \\
\text { - scientifique (ressources génetiques) } \\
\text { - culturelle (expositions, animation, visites...) } \\
\text { - economique (production...) }\end{array}$ & $\begin{array}{l}\text { "Elements de réflexion pour l'élaboration d'une } \\
\text { politique en matière de conservation fruitière" } \\
\text { AFCEV }\end{array}$ \\
\hline
\end{tabular}

Fig 2. Conservation fruitière.

\section{LA VALORISATION DE LA CONSERVATION DES VARIÉTÉS FRUITIÈRES}

Il est peut-être utile de montrer aux décideurs comment pourront être valorisés tous ces efforts concernant la recherche, l'étude et le maintien de nos anciennes variétés fruitières locales. Les valorisations attendues sont de plusieurs niveaux : il s'agira de :

- maintenir une réserve de gènes utiles aux travaux des sélectionneurs visant une diversification de la qualité des fruits, l'amélioration de la résistance aux maladies ou à certains aléas climatiques, etc; comme toute conservation de matériel végétal, la conservation des anciennes variétés fruitières est une «assurance pour l'avenir»; de quels gènes aurons-nous besoin demain? afin de répondre facilement à toute éventualité, il faut conserver une large base génétique;

- relancer dans chaque région la culture de quelques très bonnes variétés locales (dont on aura vérifié ou obtenu le bon état sanitaire); des efforts ont été réalisés par des associations et des centres de ressources génétiques pour inscrire un certain 
nombre de variétés sur les listes dites de variétés anciennes et d'amateur du CTPS (Centre technique permanent de la sélection);

- présenter au public les anciennes variétés locales et leurs usages (exposition, présentation de recettes, visites de vergers, etc);

- intéresser quelques pépiniéristes à la réalisation de plants destinés aux amateurs et aux résidents secondaires attirés par la diversité végétale locale et par des variétés particulières même si elles ne correspondent pas aux critères commerciaux actuels; - soulager les stations de recherches de la charge de collections trop importantes et difficiles à bien gérer.

\section{CONCLUSIONS}

Il a été souvent considéré comme utopique d'essayer de faire travailler en coopération des gens et des organismes, qui, bien qu'ayant des motivations différentes participent au maintien de la diversité du monde végétal. C'est cependant le pari qu'a fait l'AFCEV.

Avec l'aide du ministère de la Recherche, elle a fait un inventaire critique des différentes actions déjà engagées dans la conservation des anciennes variétés fruitières (elles concernent principalement le pommier). On peut dire :

- qu'un important travail de prospection a été réalisé sur les 2 tiers de notre pays; - malheureusement les collections d'étude dite «conservatoire» correctement conçues et entretenues sont rares; cela est dû à un manque d'expérience, de directives, et surtout de moyens;

- sur 22 vergers de collections expertisés, 7 ont obtenu le label «verger conservatoire» de l'AFCEV; 10 collections rassemblent une diversité régionale significative, mais ne respectent pas le cahier des charges de l'AFCEV (âges variables : porte-greffes différents, cahier des charges non respecté, etc). (Voir fig 3).

Il nous semble qu'il serait maintenant nécessaire que les instances nationales (ministères de la Recherche, de l'Agriculture, de l'Environnement et de la Culture) dégagent une politique de conservation. Celle proposée par l'AFCEV peut se résumer de la façon suivante :

- régionalisation des conservatoires : conserver les variétés dans leur région d'origine, où elles sont bien adaptées (cela limite les risques de perte), avec des acteurs régionaux généralement motivés pour étudier le patrimoine local;

- soutien à la constitution de collections nationales à l'abri d'attaques parasitaires majeures (ex : feu bactérien pour les arbres fruitiers à pépins, Sharka pour les arbres fruitiers à noyaux...);

- coordination nationale pour avoir une même démarche, suivant une certaine rigueur scientifique et technique, pour éviter la dispersion des moyens, pour mettre en évidence des «points noirs» (et pouvoir intervenir si certaines variétés sont menacées de disparition...).

Á ce sujet, l'AFCEV a édité un document intitulé «Connaissance et conservation de la diversité variétale chez les espèces fruitières en France : éléments pour l'élaboration d'une politique nationale».

L'AFCEV soutient les demandes de financement pour la création et la gestion de vergers conservatoires, faites par ses adhérents, notamment dans le cadre des 


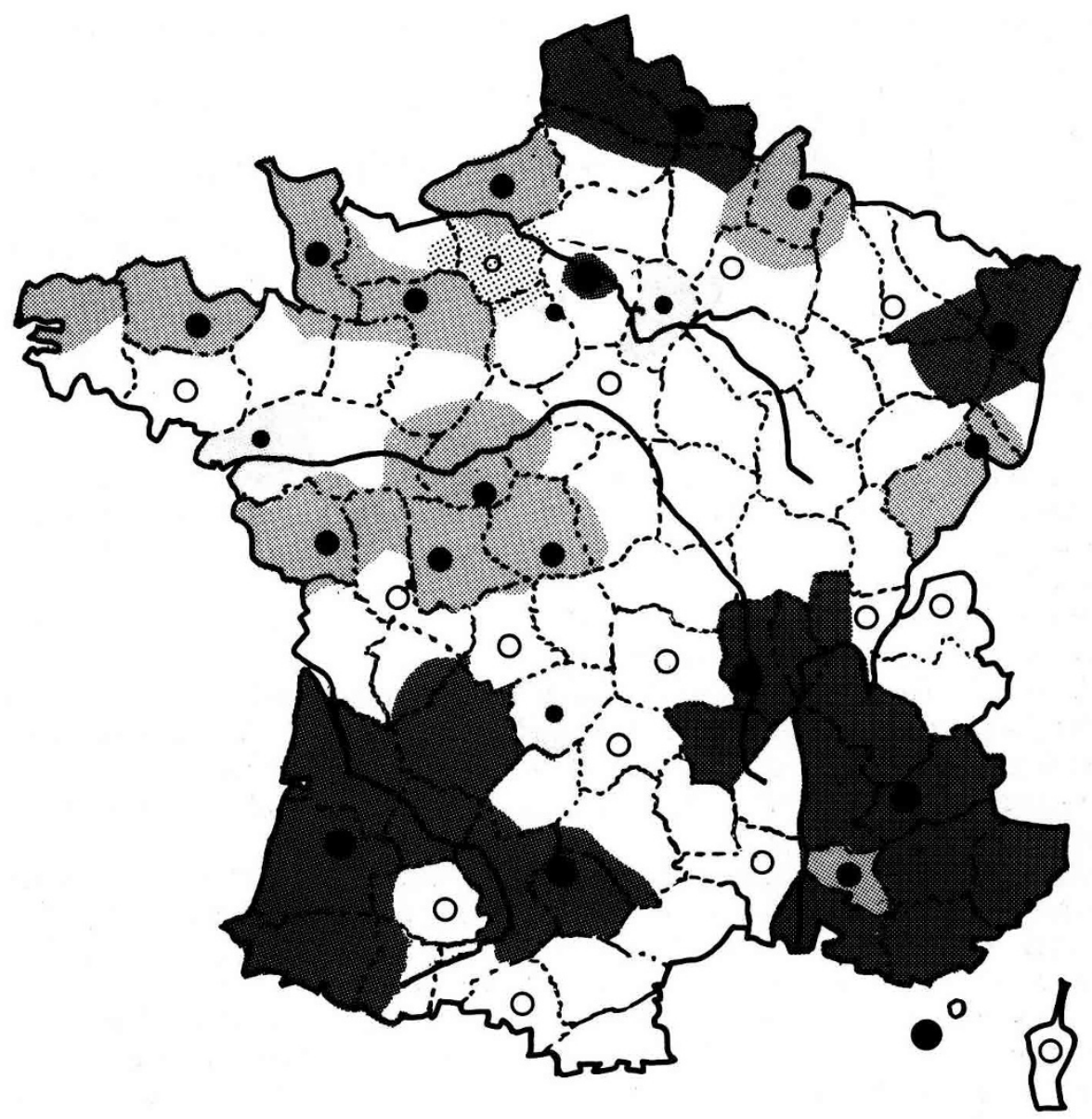

Fig 3. La carte d'implantation des conservatoires et leur aire d'action; vergers conservatoires (label AFCEV); vergers de collections fruitières réunissant une diversité variétale significative; vergers de collections fruitières de sauvegarde; $\bigcirc$ futures implantations ou absence d'implantation depuis 1987; O aire d'action : zone de prospection.

contacts de plan État-région. L'AFCEV souhaiterait des financements nationaux pour :

- la réalisation des observations systématiques et de leurs enregistrements;

- la gestion d'un fichier national situant l'ensemble du matériel identifié et conservé dans notre pays.

L'appui des ministères concernés devrait être lié à la mobilisation parallèle de moyens d'entretien régionaux et à une évaluation périodique du type de celle réalisée par l'AFCEV en 1991. Seules devraient être aidées les actions répondant au cahier des charges. 


\section{REMERCIEMENTS}

Au ministère de la Recherche, au Bureau de ressources génétiques et au Conservatoire botanique de Porquerolles, pour l'aide ayant permis l'édition des fiches descriptives variétales adaptées aux principales espèces fruitières et l'évaluation de 30 collections locales de variétés fruitières mises en place par divers organismes ou associations.

\section{RÉFÉRENCES}

AFCEV, conservatoire botanique national de Porquerolles, Fédération des parcs naturels de France, Bureau des ressources génétiques (1991)

Description pomologique des abricotiers, des amandiers, des cerisiers, des figuiers, des pêchers, des pommiers et poiriers, des pruniers

AFCEV (1991) Connaissance et conservation de la diversité variétale chez les espèces fruitières en France.

Rapport de synthèse

AFCEV (1991) Cahier des charges pour la constitution des vergers de conservation de variétés locales ou anciennes d'espèces fruitières, annexe $\mathrm{F}$

AFCEV (1991) Les collections fruitières des variétés fruitières locales et anciennes recensées en France, annexe $\mathrm{H}$

AFCEV (1991) Notice d'utilisation du logiciel pour la saisie sur support informatique des listes d'arbres en collection et des observations ou descriptions réalisées sur les variétés locales ou anciennes, annexe $\mathrm{E}$

AFCEV (1991) Les vergers conservatoires et les collections de variétés fruitières et locales en France. Présentation des comptes rendus d'expertise

Dosba F (1983) Le comportement des espèces du genre Prunus vis-à-vis du virus de la Sharka. Bull SROP, 57-66

Leroy A (1867-1879) Dictionnaire pomologique en 6 volumes (édité par l'auteur à Angers) Réédité par ADR, 04250 Turriers en 1988

Marchenay P, Lagarde MF (1986) À la recherche des variétés locales de plantes cultivées. PAGE PACA Conservatoire botanique de Porquerolles, $211 \mathrm{p}$

Marenaud C, Saunier R (1967) Thermothérapie sur pêcher. Ann Amélior Plantes 17 (1), 13-21

Marenaud C, Kéramidas C (1969) Séparation par thermothérapie du Virginia decline et de 6 autres affections virales du pommier. Ann Phytopathol 1 (4), 645-652 Massonié G (1976) Pucerons et transmission de la Sharka, INVUFLEC, 13-20 Collectif (1974) Progrès réalisés dans la connaissance de la Sharka. Bulletin de l'organisation européenne et méditerranéenne pour la protection des plantes 4, 1 
ANNEXE 1. Les caractères sur fond grisé sont les caractères minima permettant d'identifier les variétés. Ce sont ceux qui figurent sur la fiche de description des variétés d'amateur au catalogue CTPS.

\begin{tabular}{|c|c|c|c|c|c|c|}
\hline \multirow[b]{2}{*}{$\begin{array}{l}\text { Mode de conduite des arbres } \\
\text { Type de porte-greffe } \\
\text { Nombre d'années de description } \\
\text { (si fiche moyenne) ou } \\
\text { date(s) d'observation(s) }\end{array}$} & \multirow[b]{2}{*}{$\begin{array}{l}1 \\
2 \\
3\end{array}$} & 1 & 2 & \multirow[t]{2}{*}{3} & \multirow[t]{2}{*}{4} & \multirow[t]{2}{*}{5} \\
\hline & & & & & & \\
\hline Epoque de début de floraison & 4 & très précoce & précoce & moyenne & tardive & très tardive \\
\hline 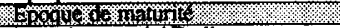 & 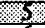 & Tres ofecoce & precoce & moxenter & 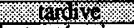 & 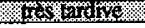 \\
\hline Date de maturité/Redhaven & 6 & & & Date de maturité & autre témoin: & \\
\hline Whoter r r & the & ins fatble & (3ibese & majeane & tistis & 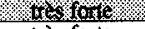 \\
\hline $\begin{array}{l}\text { Productivité } \\
\text { Epoque de débourrement foliaire } \\
\text { SENSIBILITE AUX MALADIES } \\
\text { ET ACCIDENTS }\end{array}$ & $\begin{array}{l}8 \\
9\end{array}$ & $\begin{array}{l}\text { très faible } \\
\text { très précoce }\end{array}$ & $\begin{array}{l}\text { faible } \\
\text { précoce }\end{array}$ & $\begin{array}{l}\text { moyenne } \\
\text { moyenne }\end{array}$ & $\begin{array}{l}\text { forte } \\
\text { tardif }\end{array}$ & $\begin{array}{l}\text { très forte } \\
\text { très tardif }\end{array}$ \\
\hline 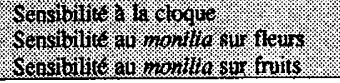 & $\frac{10}{11}$ & $\begin{array}{l}\text { non wateint } \\
\text { non alieint } \\
\text { nor ausint }\end{array}$ & & 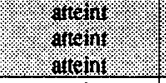 & & ins insingin: \\
\hline $\begin{array}{l}\text { Sensibilité au coryneum } \\
\text { Autres maladies }\end{array}$ & $\begin{array}{l}13 \\
14\end{array}$ & non atteint & & atteint & & tres atteint \\
\hline Caracteres dischininants & 6 & & & & & \\
\hline ARBRES - RAMEAUX & & 1 & 2 & 3 & 4 & 5 \\
\hline 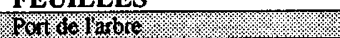 & 16. & ingso & dentidinessis & Thonzonial : & retonbant & res relouming \\
\hline $\begin{array}{l}\text { Longueur moyenne des } \\
\text { entre-nœuds du rameau mixte } \\
\text { Rapport Longueur/largeur du limbe } \\
\text { Dentelure }\end{array}$ & $\begin{array}{l}18 \\
19 \\
20\end{array}$ & $\begin{array}{l}\text { très faible } \\
\leq 5 / 20 \mathrm{~cm} \\
\text { de } 20 \text { fleurs } \\
\text { au metre } \\
\text { courts } \\
\text { très faible } \\
\leq 3 \\
\text { crénelé }\end{array}$ & $\begin{array}{c}\text { faible } \\
3<x \leq 3,5\end{array}$ & $\begin{array}{c}\text { moyenne } \\
5 \text { a } 15 / 20 \mathrm{~cm} \\
\text { de } 20 \text { a } 60 \text { fleurs } \\
\text { au mètre } \\
\text { moyens } \\
\text { moyen } \\
3,5<x \leq 4\end{array}$ & $\begin{array}{c}\text { élevé } \\
4<x \leq 4.5\end{array}$ & $\begin{array}{c}\text { très élevé } \\
\geq 15 / 20 \mathrm{~cm} \\
+ \text { de } 60 \text { fleurs } \\
\text { au mètre } \\
\text { longs } \\
\\
\text { très élevé } \\
>4,5 \\
\text { denté }\end{array}$ \\
\hline Nectuires & 21. & dosents & 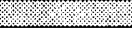 & circulaires & 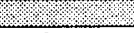 & rent formes \\
\hline Stipules & 22 & $\begin{array}{l}\text { très courts } \\
<9 \mathrm{~mm}\end{array}$ & $\begin{array}{c}\text { courts } \\
9-12 \mathrm{~mm}\end{array}$ & $\begin{array}{c}\text { moyens } \\
12-15 \mathrm{~mm}\end{array}$ & $\begin{array}{c}\text { longs } \\
15-20 \mathrm{~mm}\end{array}$ & $\begin{array}{l}\text { très longs } \\
>20 \mathrm{~mm}\end{array}$ \\
\hline Caracteres discriminants & 23. & & & & & \\
\hline FLEURS & & & & & & \\
\hline Forme de la fleur & 24 & Campanules & & & $\sqrt{10}$ & Rosacée \\
\hline $\begin{array}{l}\text { Taille du pétale } \\
\text { Couleur de la paroi inteme } \\
\text { du calice }\end{array}$ & $\begin{array}{l}25 \\
26\end{array}$ & $\begin{array}{c}\text { petit } \\
\text { blanc crème }\end{array}$ & & moyen & & $\begin{array}{l}\text { grand } \\
\text { orangé }\end{array}$ \\
\hline
\end{tabular}




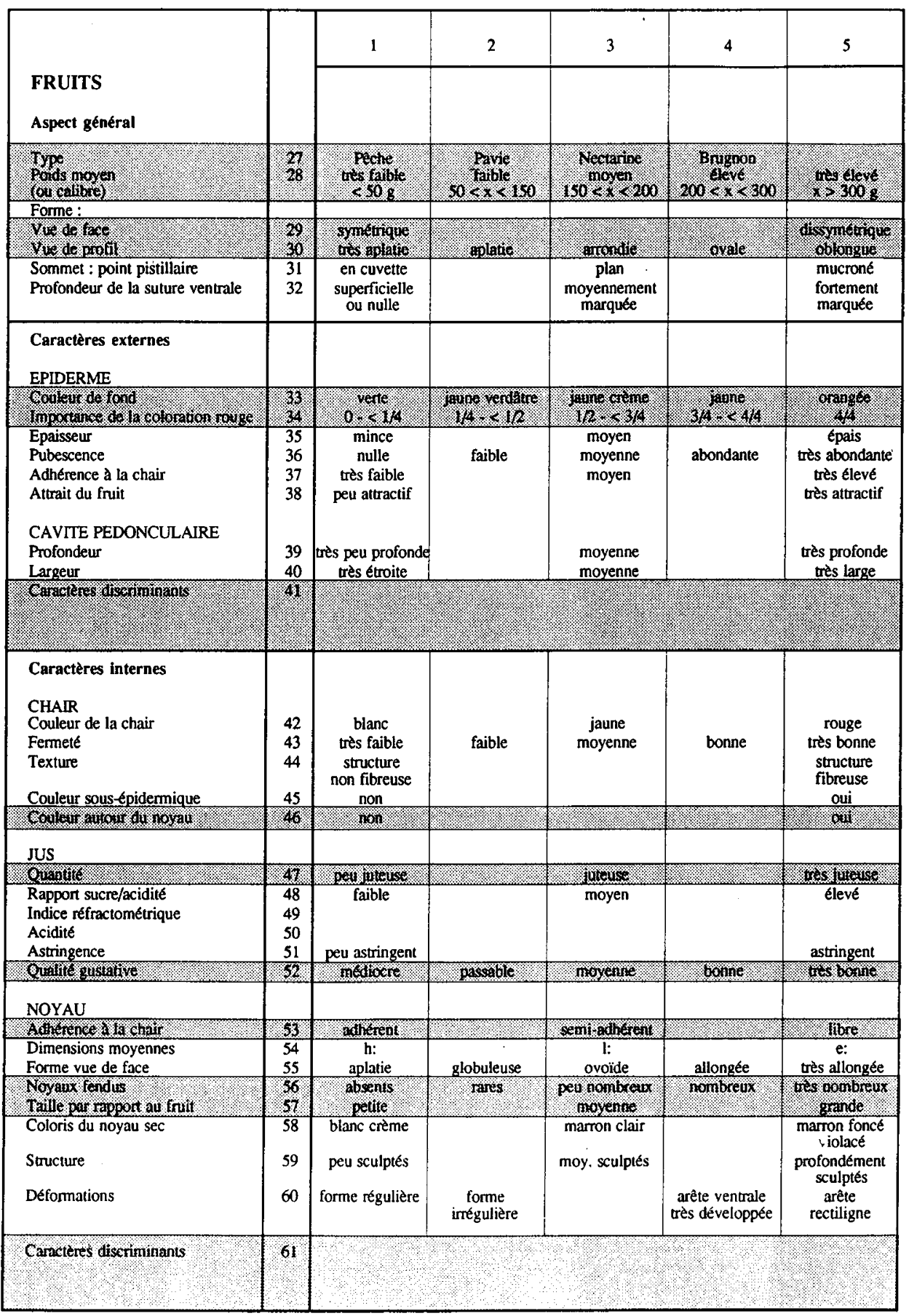




\section{ANNEXE 2. Extrait du guide de description pomologique de la fiche «pêcher»}

Notes attribuées

$\begin{array}{lllll}1 & 2 & 3 & 4 & 5\end{array}$

Témoins pêchers

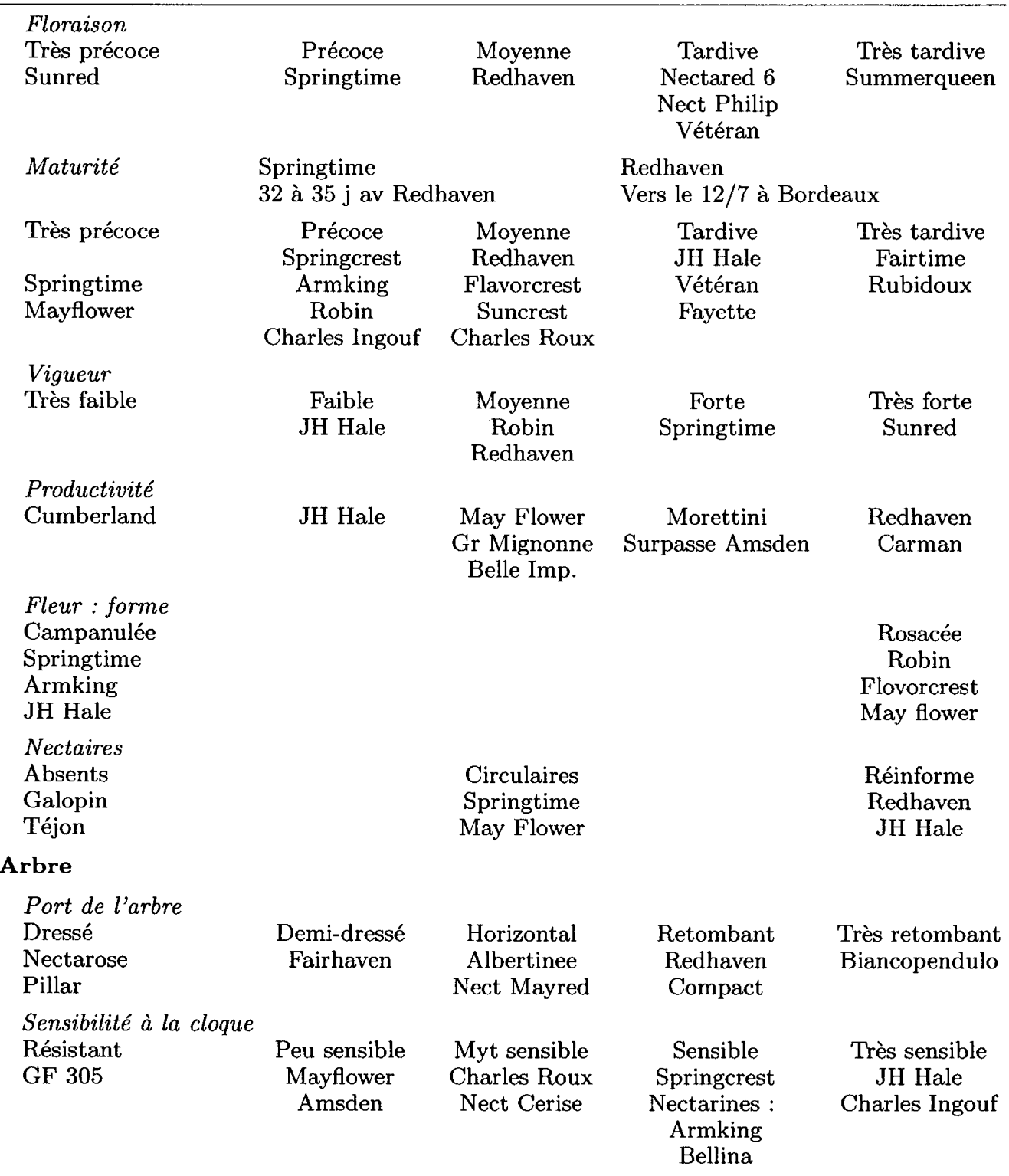


Annexe 2 (suite)

\section{Fruit}

Calibre du fruit

Très petit

Nect Cerise

$\begin{array}{cc}\text { Petit } & \text { Moyen } \\ \text { Springtime } & \begin{array}{c}\text { Sunhaven } \\ \text { Springcrest }\end{array}\end{array}$

Gros

Loring

Redtop

Forme du fruit

Très aplatie

Aplatie

Arrondie

Platicarpa

$$
\text { Robin }
$$

Redwing

Springcrest

Cavité pédonculaire : profondeur

Superficielle

Peu profond

Moyenne

Profonde

Très profonde

Robin

Southland

Cavité pédonculaire : largeur

Très étroite

$$
\text { Étroite }
$$

Moyenne

Large

Robin

Très gros

JH Hale

Redhaven

Forme du sommet: (/ plan tangent)

En cuvette

Au niveau du plan tangent

Amsden

Redhaven

Robin

Couleur superficielle

Verte

Jaune verdâtre

Jaune crème

Ruberrina

Springtime

JH Hale

Jaune

Redhaven

Oblongue

Elberta

Adhérence de l'épiderme à la chair

Très faible

Faible

Madame Girerd

Redhaven

May Flower

Galande

Moyenne

Nect Early

Sungrand

Forte

Pavie Babygold 5

Roger Louis

Très large

(>30 mm)

Late Elberta

Mucronné

Téton de Vénus

Springtime

Jaune orange

Redtop

Très forte

Pavie Vivian

Pavie Saint

Jacques Jaune

Pubescence

Nulle

Faible

Moyenne

Préc de Hale

Brugnons

Genard

Abondante

Sanguine

Vineuse

Très abondante

Triumph

Profondeur de la suture ventrale

Superficielle

Moyennement

marquée

Marquée

ou nulle

Importance de la coloration rouge de l'épiderme
Très faible $\quad$ Faible

Vétéran

Amsden

Redhaven

Forte

Suncrest

Très forte

Red top

Épaisseur de l'épiderme

Mince

Moyenne

Hélène Turc

Précoce

de Hale

Epaisse

Carman 
Annexe 2 (suite)

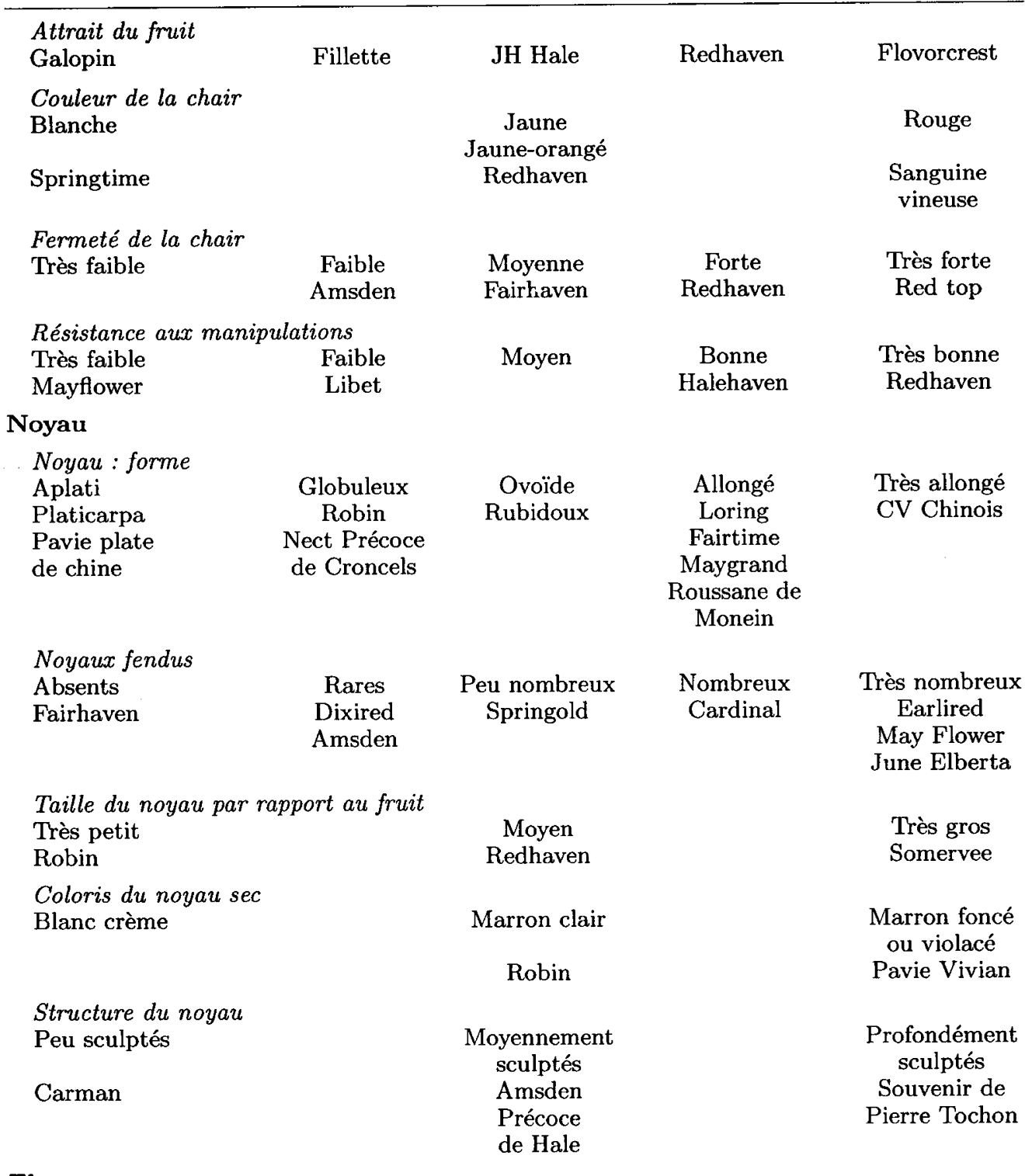

Fleur

Couleur de la fleur

Blanc

Rose très pâle Rose pâle

Rose moyen

Rose foncé ou rouge

Biancopendulo

Pavie Cardinal

Charles Ingouf

Nect Fuzalode

Red Flower

Peacer 
Annexe 2 (suite)

Préc de Hale

Pavie Vivian

Berroni

Taille du pétale

Petit

Rosacées

Campanulées

$\geqslant 9$

Moyen

17-18

10-11

Feuille

Débourrement foliaire

Très précoce

Nect Sunred

Early Elberta

Précoce Moyen

Springtime Redhaven

Moyen

Nect Early

Sungrand

Mountaingold

Stipules : longueur

Très courts

Longueur du pétiole

Très courts

$<8 \mathrm{~mm}$
Courts
Redhaven

Courts

8-10 mm

Redhaven
Moyens

Robin

Moyens

$10 \mathrm{~mm}$

Genadix 7
Tardif

Genadix 7

Elevé

Springtime

Longs

Dixired

Longs

10-12 mm

Pavie Andross
Grand $\geqslant 19 \mathrm{~mm}$

$\geqslant 12 \mathrm{~mm}$

Très tardif

Nect Philp

Reine des

Vergers

Très élevé

Très longs

Très longs

$>12 \mathrm{~mm}$ 